\title{
The pieces of the caries puzzle align
}

Nigel Pitts

Faculty of Dentistry, Oral \& Craniofacial Sciences, King's College London, UK

The BDJ Upfront section includes editorials, letters, news, book reviews and interviews. Please direct your correspondence to the News Editor,

Kate Quinlan at k.quinlan@nature.com. Press releases or articles may be edited, and should include a colour photograph if possible.

$\mathrm{D}$ ental caries is still a major problem, both locally and globally. Caries is the most prevalent noncommunicable disease (NCD) globally and affects all age groups across the life course. Yet, despite the accepted scientific evidence that caries creates very significant personal, societal, and economic burdens across the world and that caries presents the largest numerical impact across oral health, the disease has typically been invisible in 'upstream' global health policies. ${ }^{1}$ This situation appears to be paradoxical, as the burdens and pain caused by caries and cavities are preventable and addressing the risk factors for cavities can also reduce other NCDs and improve general health. ${ }^{1}$

There has also been a stubborn disconnect between accepted international evidence on how best to prevent and manage caries and the care delivered and paid for at the 'downstream' level of dental practice. In 2004 the question was asked 'Are we ready to move from operative to non-operative/preventive treatment of dental caries in clinical practice?'2 Unfortunately, in mainstream practice in many countries, the answer over decades has been 'No.' However, there is now both international agreement that the dental profession should be delivering minimally interventive caries care $^{1}$ and consensus on how to move the related evidence into practice. ${ }^{3}$ An unforeseen opportunity has come from the tragic COVID19 pandemic as there is now an appetite to avoid aerosols, follow the evidence, focus on prevention and contemplate significant system changes where there are health benefits. ${ }^{3}$

To make progress with caries control we need movement in both of these areas, as well as aligning the other key pieces of the Caries Puzzle. ${ }^{1}$ The Alliance for a Cavity Free Future (ACFF) Caries Puzzle model includes the eight key elements that need to be brought together to achieve and maintain a cavity-free future. These are: nutrition (sugar); education

and behaviour change; cariology (including fluoride); public health (upstream policy and inequalities); clinical practice (CariesCare international 4D); ICCMS caries management (education, research and dental public health); action for health professions; action for public, patients and others. This alignment of caries puzzle pieces needs to happen 'Glocally'.

'Glocal' is an approach used by ACFF in which global evidence and consensus are considered at the local level to enable appropriate adaptation and implementation at the country or more local level. Local adaptations of the relative 'sizes' of the individual puzzle pieces need to be made to

\section{'It now seems possible to embark on global and local policies to reduce cavities'}

meet the local situations in terms of specific care needs, culture, priorities and economy. It is important to appreciate that alignment is also needed between 'upstream' global policies, the 'country' level and the 'downstream' reality where dental teams assisted by other healthcare professions can play their part in preventing and controlling caries across the lifecourse.

The good news is that, at last, building on the developing global consensus amongst the dental, scientific, and public health communities, we now appear to be at a unique point in time when many interests and opportunities align. It now seems possible to embark on global and local policies to reduce cavities and to improve not only oral health but also NCDs, as well as wider health and wellbeing. ${ }^{1}$

Clarity around this opportunity has been helped through the creation of a taskforce convened by the ACFF last year. These 35 individuals have a broad range of scientific, specialist and public health expertise across a diverse range of disciplines from a total of 20 countries. They have created consensus policy recommendations which, if implemented, will help 'Make Cavities History.'

The timing of these recommendations is fortuitous as, in recent months, we have had significant progress at the global level from the WHO seeking to renew political commitment to oral health as part of WHO's NCDs and Universal Health Coverage agendas. In January 2021 the WHO’s 148th Executive Board considered and approved a draft WHO 2021 Oral Health Resolution ${ }^{4}$ (the first on oral health in 14 years). The Resolution has powerful strategies for improving oral health and health seeking 'to reorient the traditional 